\title{
Resilience to aging is a heterogeneous characteristic de- fined by physical stressors
}

\author{
Haoyi Lei ${ }^{\mathrm{a}}$, Derek M. Huffman ${ }^{\mathrm{b}}$, Adam B. Salmon ${ }^{\mathrm{c}}$, Nathan K. LeBrasseur ${ }^{\mathrm{d}}$, Christy Carter ${ }^{\mathrm{e}}$, Arlan \\ Richardson $^{\mathrm{f}}$, Steve Austad ${ }^{\mathrm{g}}$, Warren Ladiges ${ }^{\mathrm{a}, *}$
}

${ }^{a}$ Department of Comparative Medicine, School of Medicine, University of Washington, Seattle, WA, USA.

${ }^{b}$ Departments of Molecular Pharmacology, Medicine and Institute for Aging Research, Albert Einstein College of Medicine, Bronx, NY, USA.

'San Antonio Sam and Ann Barshop Institute for Longevity and Aging Studies and Department of Molecular Medicine, The University of Texas Health Science Center at San Antonio, Department of Molecular Medicine, South Texas Veterans Health Care System, Geriatric Research Education and Clinical Center, San Antonio, TX, USA.

${ }^{d}$ Department of Physical Medicine \& Rehabilitation, Mayo Clinic, Rochester, MN, USA.

${ }^{e}$ Department of Medicine, Division of Gerontology, Geriatrics and Palliative Care, School of Medicine, University of Alabama Birmingham, Birmingham, AL, USA.

${ }^{f}$ Department of Biochemistry \& Molecular Biology, University of Oklahoma Health Science Center. Oklahoma City VA Medical Center, Oklahoma City, OK, USA.

${ }^{g}$ Department of Biology, University of Alabama Birmingham, Birmingham, AL, USA.

\begin{abstract}
Physical resilience, the capacity to respond to and recover from a stressful event, declines with advancing age. Individuals respond differently to physical stressors across their lifespans. While the biological underpinnings of resilience remain unclear, a plausible determinant is the capacity of an individual's cellular and molecular levels to return to homeostasis after a physical challenge. Impaired resilience may not only be a consequence of aging but could also be a contributing factor to the aging process. Therefore, resilience at relatively younger ages could be predictive of future health and lifespan. By utilizing standardized physical challenges and measuring stress response patterns, the relative resilience of individuals can be quantified and classified. Current preclinical research suggests that several physical stressors could be used to measure resilience in clinical aging studies. A mechanistic understanding of why some individuals are more resilient to physical stressors than others could help identify protective factors and therapeutic ways to promote healthy aging.

Keywords: Physical resilience to aging, physical stressors, heterogeneity, stress response patterns, healthy aging, therapeutic resilience
\end{abstract}

\section{Introduction}

Resilience is the ability to return to homeostasis after a physically stressful event [1]. An all-encompassing characteristic influencing resilience is the inherent individual variation in response to a specific stressor [2]. It is a unique individual trait maintained throughout life but

\footnotetext{
* Correspondence author: Warren Ladiges
}

Mailing address: Department of Comparative Medicine, School of Medicine, University of Washington, Seattle, WA 98195, USA.

Email: wladiges@uw.edu

Received: 19 January 2022 / Accepted: 24 January 2022 not necessarily to the same degree from young to old. Therefore, the ability to document resilience at a younger age would likely predict that an individual would be more resilient to physical stress at an older age, compared to an individual that was determined to be less resilient at a younger age. A deeper understanding as to why some individuals maintain or regain function following an insult, while others do not, may help to characterize protective factors that can be engaged to promote resilience and healthy aging.

A major issue is defining specifics for responses to physical challenges, including how to measure resilience at a younger age and determining the optimal age, mode, and intensity of stressor(s) to reliably discriminate resilience in mammals. One approach is the development of physical stressors and the characterization of stress response patterns (SRPs). An SRP would encompass multiple factors 
involved in responding to a specific stressor. In order to characterize SRPs, a better understanding of resilience at the molecular, cellular, and organismal levels is needed. In addition, interventions such as pharmaceuticals, diet, and exercise most likely can alter the course of SRPs (Figure 1). Therefore, investigations into the molecular pathways incited by cells in a particular tissue and organ in response to an insult would be of merit.

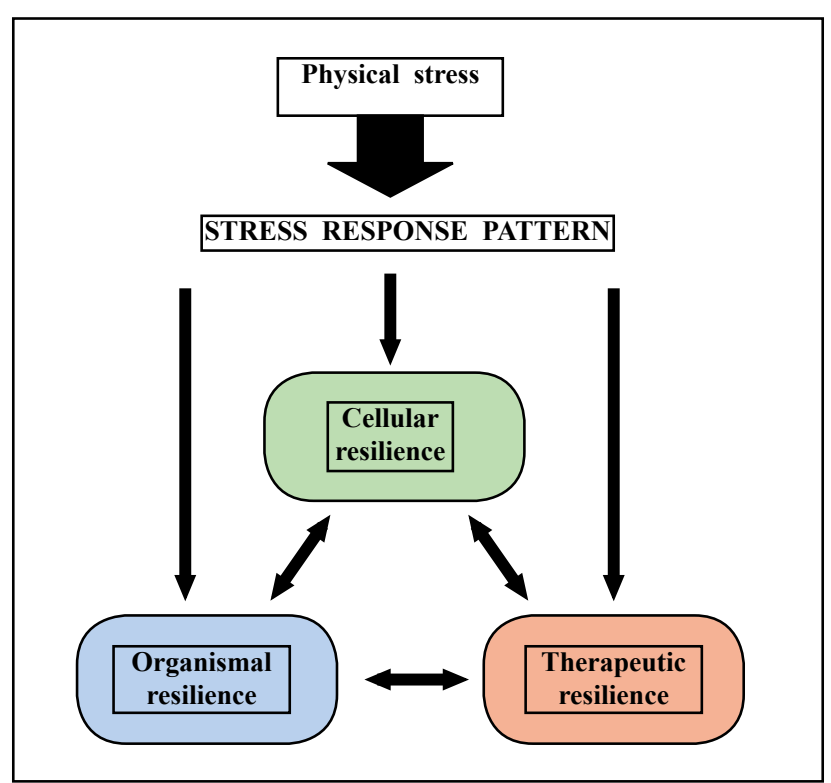

Figure 1. The stress response pattern triggered by a physical stressor drives resilience at the cellular and organismal levels in an interactive manner that can be influenced by therapeutic intervention.

\section{Factors influencing resilience and the stress re- sponse pattern}

At a young age, resilience is greater with a wider ability to return to the original state. With increasing age, gradual deficits in resilience begin to emerge that impair the ability to completely return to homeostasis. Examples of changes with age might include baseline differences before the challenge, differences in acute response, and differences in the return to homeostasis. A plethora of external and internal factors influence the timeframe and rate of resilience loss across the lifespan. External factors include the type of stress which determines the tissues, cells, and organs targeted, the severity of the stressful event, and the duration and frequency of the stress. Internal factors include the strength and speed of the return to homeostasis, the cellular and molecular pathways activated, organismal changes or comorbidities, and the existence and exacerbation of internal stressors such as oxidative stress, inflammation and cellular senescence [3].

The type of physical stress has a significant effect on the SRPs, and which tissues are likely affected by SRPs. Stressors such as surgery, anesthesia, hyperthermia, hypoxia, chemotherapy, trauma, infections, toxins, and sleep disruptions all have unique characteristics that target specific tissues and cells. In addition, the severity and duration of each stressor can exacerbate or overwhelm the re- sponse. Multiple interactive SRPs can also be carried out by different cell types and multiple organs depending on the degree of macromolecular damage. For example, sleep deprivation generates neuropathological events related to cognitive dysfunction but also creates metabolic disturbances, even on a short-term basis [4]. Long-term sleep deprivation causes more severe adverse effects putting more strain on SRPs to return an individual to homeostasis.

There are numerous cellular and molecular pathways within organ systems that are programmed to respond to stress in different ways. The immune system is a good example. Inflammation operates from programmed pathways, such as the monocyte chemotactic protein-1 (MCP1) signaling pathway or the inflammasome [5]. Similarly, wound healing is highly dependent on the response of fibroblasts. Antibody and T-cell responses are put to the test with the introduction of vaccines, infectious agents, or toxins. Other pathways are just as important. Mitophagy is activated by oxidative stress in cells to remove damaged mitochondria [6]. Mitochondria produce reactive oxygen species (ROS) during oxidative phosphorylation. Normal levels of ROS help maintain cellular behavior, but excess levels can cause molecular damage and oxidative stress. Another pathway involved in resilience is senescence [7]. Senescence pathways are evident in the majority of cell types, including fibroblasts, macrophages, and endothelial cells, with variability in cytokine purges. Senescent cells accumulate with advancing age in response to various stressors, including DNA damage and proliferative exhaustion. However, cellular stress and damage can trigger premature senescence as a means to suppress the growth of damaged cells. Environmental stressors can lead to DNA damage, which causes cell cycle arrest at the G1/S checkpoint [8]. A final process for discussion is proteostasis. This process is largely responsible for the maintenance of a balanced proteome, and it regulates correct protein synthesis while also removing misfolded, damaged proteins. Under cellular stress or with aging, the functionality of the proteostasis network declines, leading to the accumulation of protein aggregates. This is particularly common in neurodegenerative diseases, such as Alzheimer's Disease and Parkinson's Disease [9].

Differences in resilience may be caused by both genetic and non-genetic regulation. For example, variation among individuals regarding the molecular pathways discussed above may be determined in part by the genetic composition of each individual. However, non-genetic regulation, including epigenetic factors, is also likely to play a role in these differences. Throughout the mammalian lifespan, epigenetic changes influence gene expression levels. For example, histone deacetylase (HDAC) inhibitors have been shown to enhance healthy aging [10]. Though the exact process through which they extend lifespan has not been confirmed, HDAC inhibitors prevent de-acetylation of histones, thereby increasing gene expression [11]. This may help reverse the decreased expression of genes involved in maintaining homeostasis seen with aging.

A final factor likely influencing resilience is the speed of 
recovery from physical stress, which is a novel measure of latent aging. This is influenced by the capacity to respond and depends on how much reserve there is to respond, as well as the actual strength of the response. Indeed, resilience is a dynamic process, and the speed of recovery can be determined and quantified depending on the stressor. For example, the response to and recovery from a nonlethal dose of the chemotherapeutic drug cyclophosphamide, which targets myeloid precursor cells, can easily be determined by a simple white blood cell count measuring the acute decrease with challenge and gradual recovery of cells [12].

\section{The stress response pattern reflects variability with increasing age}

The individual variability in resilience with increasing age is also the result of variation in internal factors affecting the stress response. A recent publication studied drivers of this variability by profiling fibroblast cultures from young and old mice that have different reprogramming efficiencies [13]. This approach revealed that fibroblast cultures from old mice contain activated fibroblasts (myofibroblasts) that secrete inflammatory cytokines, including tumor necrosis factor- $\alpha$ (TNF- $\alpha)$. Notably, old mice exhibited variability in wound healing rate. Single-cell RNA-sequencing analysis identified distinct subpopulations of fibroblasts with different cytokine expression and signaling in the wounds of old mice with slow versus fast healing rates. Hence, a shift in fibroblast composition, and the ratio of inflammatory cytokines that they secrete, may drive the variability between mice and influence wound healing rate. This variability may reflect distinct stochastic aging trajectories between individuals and could help in developing personalized strategies to improve wound healing with increasing age.

The physical challenge can measure response patterns in a phenotypic manner and can be used to identify phenotypic variability to a specific challenge. Any number of stressors can be tested in aging mice, but those that have translational relevance would provide information about the applicability to clinical studies. These stressors can then be developed to define SRPs in mice that identify phenotypic variability with potential translational targets. Several relevant stressors include 1) Endotoxin, inducing a serum cytokine response; 2) Pneumococcal conjugate vaccine 13, inducing a serum antibody response; 3) Isoflurane, measured by anesthetic recovery time; 4) cyclophosphamide, measured by peripheral blood neutrophil response; 5) Sleep deprivation, measured by the degree of cognitive impairment; and 6) Surgical ear punch biopsy, measured by wound healing rate response. The phenotypic variability of these stress responses can be used to quantify and even classify subjects over a range of high responders and low responders in relation to healthspan (Figure 2) and lifespan. However, the extent to which resilience or vulnerability to one form of stressor necessarily implicates a similar response to other stressors remains unclear and

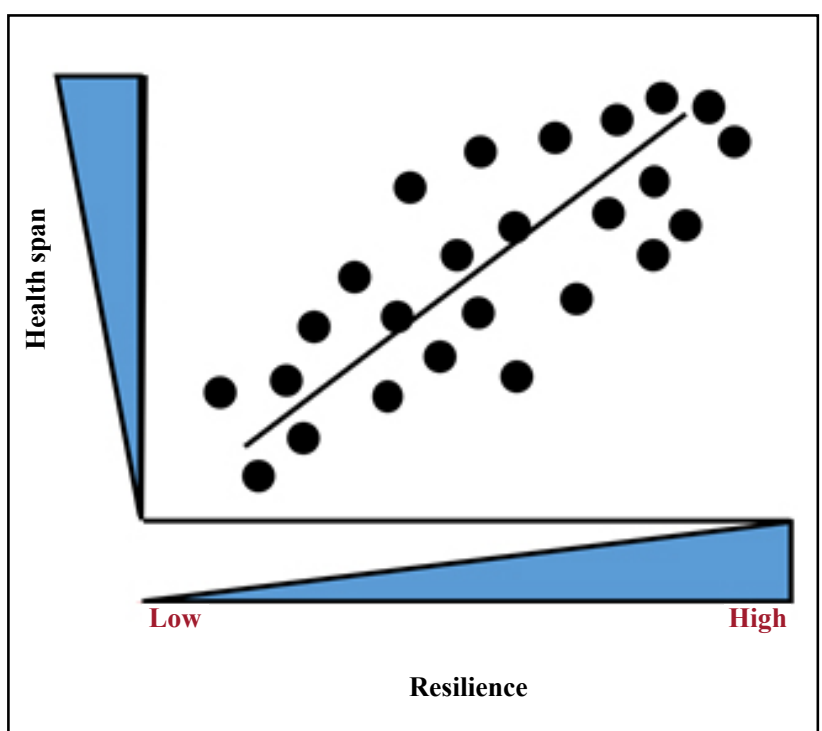

Figure 2. Measuring the stress response patterns to specific physical stressors can classify individuals over an increasing range from low to high resilience in relation to healthy aging.

could reflect in part, heterogeneity of resilience among different organ-systems in the same animal. Since single stressors most likely are limited to a particular target, combinations of stressors would be of interest to study.

Evidence of the genetic basis of SRPs has not yet been widely reported. In order to understand the heterogeneity of resilience, molecular mechanisms associated with the stress response patterns must be identified. Multiomics profiling of cells from high response individuals and low response individuals can provide vital information on the variation of genetic patterns. Single cell RNA-Seq is a procedure for cellular analysis that can be used to analyze the quantity and presence of RNA at any given moment. This information is used to determine which genes are turned on or off and degree of expression at the level of individual tissues or single cells. Comparing the transcripts of cells from resilient and non-resilient individuals could shed light on the role of genetics in stress responses and why certain individuals are more resilient to stressors than others [14].

\section{Conclusion}

The ability of an organism to respond to and recover from a physical stressor is defined as physical resilience. With increasing age, physical resilience declines thereby increasing vulnerability to stress. Thus, measuring resilience at a relatively young age through physical challenges and SRPs could help classify relatively resilient and nonresilient individuals and predict health trajectories. Once the molecular mechanisms that contribute to individual heterogeneity have been identified, they can be used to develop interventions focused on optimizing resilience with increasing age for each individual. Some may need little intervention, while others may need a more aggressive intervention approach, which could include therapeutics and lifestyle changes. 


\section{Declarations}

Availability of data and materials: Not applicable.

Financial support and sponsorship: P30AG038072 (DH), R01 AG057429 (DH), R01 AG057381 (WL), R01 AG057434 (SA), R01 AG053832 (NL), P01 AG062413 (NL), R01 AG057431 (AS), R01 AG057424 (AR), Senior Career Research Award 1IK6BX005238 (AR) from the Department of Veterans Affairs.

Conflicts of interest: Adam B. Salmon, Christy Carter, and Warren Ladiges are members of the Editorial Board of Aging Pathobiology and Therapeutics. All authors declare no conflict of interest and were not involved in the journal's review or decisions related to this manuscript.

Ethical approval and consent to participate: Not applicable.

\section{References}

1. Kirkland J L, Stout M B, Sierra F. Resilience in aging mice. Journals of Gerontology Series A: Biomedical Sciences and Medical Sciences, 2016, 71(11): 1407-1414.

2. Schorr A, Carter C, Ladiges W. The potential use of physical resilience to predict healthy aging. Pathobiology of Aging \& Age-related Diseases, 2018, 8(1): 1403844.

3. LeBrasseur N K. Physical resilience: opportunities and challenges in translation. The Journals of Gerontology: Series A, 2017, 72(7): 978-979.

4. Wu J, Dou Y, Ladiges W C. Adverse neurological effects of short-term sleep deprivation in aging mice are prevented by SS31 peptide. Clocks \& sleep, 2020, 2(3): 325333.
5. Yousefzadeh M J, Schafer M J, Noren Hooten N, et al. Circulating levels of monocyte chemoattractant protein- 1 as a potential measure of biological age in mice and frailty in humans. Aging cell, 2018, 17(2): e12706.

6. Shefa U, Jeong N Y, Song I O, et al. Mitophagy links oxidative stress conditions and neurodegenerative diseases. Neural regeneration research, 2019, 14(5): 749.

7. Kirkland J L, Tchkonia T, Zhu Y, et al. The clinical potential of senolytic drugs. Journal of the American Geriatrics Society, 2017, 65(10): 2297-2301.

8. Raghuram G V, Mishra P K. Stress induced premature senescence: a new culprit in ovarian tumorigenesis?. The Indian journal of medical research, 2014, 140(Suppl 1): S120.

9. Hipp M S, Kasturi P, Hartl F U. The proteostasis network and its decline in ageing. Nature reviews Molecular cell biology, 2019, 20(7): 421-435.

10. Brunet A, Berger S L. Epigenetics of aging and aging-related disease. Journals of Gerontology Series A: Biomedical Sciences and Medical Sciences, 2014, 69(Suppl_1): S17-S20.

11. McIntyre R L, Daniels E G, Molenaars M, et al. From molecular promise to preclinical results: HDAC inhibitors in the race for healthy aging drugs. EMBO molecular medicine, 2019, 11(9): e9854.

12. Zhu L, Dou Y, Bjorner M, et al. Development of a cyclophosphamide stress test to predict resilience to aging in mice. GeroScience, 2020, 42(6): 1675-1683.

13. Mahmoudi S, Mancini E, Xu L, et al. Heterogeneity in old fibroblasts is linked to variability in reprogramming and wound healing. Nature, 2019, 574(7779): 553-558.

14. Wang X, Liu Q, Zhang B. Leveraging the complementary nature of RNA-Seq and shotgun proteomics data. Proteomics, 2014, 14(23-24): 2676-2687.

Cite this article as: Lei H Y, Huffman M D, Salmon A B, et al. Resilience to aging is a heterogeneous characteristic defined by physical stressors[J]. Aging Pathobiology and Therapeutics, 2022, 4(1): 19-22. 